\title{
Development of a comprehensive database of scattering environmental conditions and simulation constraints for offshore wind turbines
}

\author{
Clemens Hübler, Cristian Guillermo Gebhardt, and Raimund Rolfes \\ Institute of Structural Analysis, Leibniz Universität Hannover, Appelstr. 9a, 30167 Hanover, Germany \\ Correspondence to: Clemens Hübler (c.huebler@isd.uni-hannover.de) \\ Received: 17 May 2017 - Discussion started: 8 June 2017 \\ Revised: 4 September 2017 - Accepted: 23 September 2017 - Published: 26 October 2017
}

\begin{abstract}
For the design and optimisation of offshore wind turbines, the knowledge of realistic environmental conditions and utilisation of well-founded simulation constraints is very important, as both influence the structural behaviour and power output in numerical simulations. However, real high-quality data, especially for research purposes, are scarcely available. This is why, in this work, a comprehensive database of 13 environmental conditions at wind turbine locations in the North and Baltic Sea is derived using data of the FINO research platforms. For simulation constraints, like the simulation length and the time of initial simulation transients, wellfounded recommendations in the literature are also rare. Nevertheless, it is known that the choice of simulation lengths and times of initial transients fundamentally affects the quality and computing time of simulations. For this reason, studies of convergence for both parameters are conducted to determine adequate values depending on the type of substructure, the wind speed, and the considered loading (fatigue or ultimate). As the main purpose of both the database and the simulation constraints is to compromise realistic data for probabilistic design approaches and to serve as a guidance for further studies in order to enable more realistic and accurate simulations, all results are freely available and easy to apply.
\end{abstract}

\section{Introduction}

Although the share of offshore wind energy in overall energy production has been steadily growing over the last years, the cost of offshore wind energy is still high compared to other renewable energies (Kost et al., 2013). In order to achieve potential cost reductions of about $30 \%$ in the next 10 years (Prognos AG and Fichtner, 2013), a realistic and accurate simulation of offshore wind turbines and their substructures is beneficial. On the one hand, for realistic simulations, the knowledge of scattering environmental conditions is a central point. In this context, scattering conditions are non-constant parameters that exhibit stochastic variations and aleatoric uncertainties, and therefore should be modelled as statistically distributed. On the other hand, carefully chosen simulation constraints, like the simulation length or the time of initial transients, are essential to obtain accurate results. Here, the simulation length is defined as the usable time for the post- processing. The time of initial transients is the time that is removed from each simulation to exclude initial transients resulting from starting a calculation with a set of initial turbine conditions (like rotor speed). Simulation length plus initial transient time make up the overall length.

Regarding the first point, current guidelines (IEC, 2009) already define that simulations should mirror the changing environmental conditions at the precise site of a wind turbine. However, for academic research, real site data are rarely available, and, even for industrial purposes, data quality might be poor for some parameters or long-term data might be missing. As a result, various research projects have characterised environmental conditions at specific sites or entire areas and published statistical distributions as a reference. Probably the most frequently used example is the UPWIND design basis (Fischer et al., 2010). Further examples are the work of Stewart et al. (2015), the PSA-OWT project (Hansen et al., 2015), and the investigations by Häfele et al. 
(2017). All these reference conditions have some limitations. The design basis of Stewart et al. (2015) is only for deepwater sites off the coasts of the United States of America. The wave state of deep-water sites is not comparable to shallowwater conditions in the North Sea, as significant wave heights generally increase with the water depth (Hansen et al., 2015). Additionally, wind speeds are not measured at hub height and therefore have to be extrapolated, which increases uncertainties. For the UPWIND design basis, the wind speed is just given at a reference height of $10 \mathrm{~m}$ and not at hub height as well. Furthermore, no statistical distributions for conditional parameters (e.g. the wave height $H_{\mathrm{s}}$ depends on the wind speed $v_{\mathrm{s}}$ ) are given, only scatter plots. In the PSA-OWT project, data of the research platform FINO1 in the North Sea are used. Here, the wind speed is measured at hub height, but shadow effects can occur if sensors are positioned behind the measuring mast. Häfele et al. (2017) use data of the research platform FINO3, which has several sensors at each height to reduce shadow effects. However, only five environmental parameters (wind speed and direction, wave height, period and direction) are analysed, and the data period is only 5 years. Hence, the need for a comprehensive database, covering several sites and the most important parameters, becomes obvious in order to enable future research that is based on realistic data. Missing conditions are, for example, the turbulence intensity, the wind shear, or ocean currents.

As to the second point, simulation constraints are frequently chosen based on experience, literature values, or recommendations in current standards. However, considering the simulation length and time of initial transients, recommendations in the guidelines are mainly fairly vague (GL, 2012; IEC, 2009). Simulation lengths of $10 \mathrm{~min}$ for fatigue calculations (FLS), and $1 \mathrm{~h}$ or less for ultimate loads (ULS) are frequently recommended. For the initial transients, it is advised to discard lengths of $5 \mathrm{~s}$ or more. Literature values partly differ significantly. To reduce the effects of initial transients, the first 20,30, or $60 \mathrm{~s}$ are discarded, for example (Vemula et al., 2010; Jonkman and Musial, 2010; Hübler et al., 2017), and simulation lengths of $10 \mathrm{~min}$ and $1 \mathrm{~h}$ are common practice (Jonkman and Musial, 2010; Popko et al., 2012; Cheng, 2002). However, longer simulation lengths are partly used as well, especially in the oil and gas industry or for floating substructures (DNV, 2013). Still, all these recommendations are not underpinned with detailed analyses. For floating offshore wind turbines, such investigations were conducted for the simulation length by Stewart et al. (2015), Stewart et al. (2013) and Haid et al. (2013). It is shown that simulation lengths of $10 \mathrm{~min}$ are sufficient for ULS and FLS loads. The observation that ULS and FLS loads tend to be higher for longer simulations is not for physical reasons but due to unclosed cycles in the rainflow counting for the FLS case and a result of the averaging technique in the case of ULS loads. Both can be handled by adapting the algorithms. Concerning the time of initial transients, Haid et al. (2013) recommend $60 \mathrm{~s}$ and the utilisation of initial conditions. This recommendation is based on an analysis which has not been further specified. For a jacket foundation, Zwick and Muskulus (2015) conducted a study investigating lengths of simulations and initial transients and also concluded that $10 \mathrm{~min}$ is sufficient, as long as $10 \mathrm{~min}$ time series are merged before the rainflow counting is applied. The required time of initial transients is determined by checking the rotor speed to reach a steady state. However, the initial conditions are not applied, and a steady speed does not guarantee that all transients are damped out. Therefore, the need for well-founded guidance on simulation lengths and times of initial transients for bottom-fixed substructures becomes clear. For the simulation length, useful preliminary work is available, but it is limited to jacket substructures. Concerning initial transients, extensive studies are rare and do not concentrate on the convergence of the relevant loads (FLS and ULS). Furthermore, scattering environmental conditions are not taken into account. This is a simplification especially in the case of the initial transients, as this variation might lead to more pronounced resonance effects (e.g. rarely occurring low wave peak periods that are close to the natural frequency of the structure; see Sect. 2.4) and therefore to more pronounced initial transients.

After all, the listed shortcoming in state-of-the-art modelling assistance motivated the current work that focuses on the following aspects:

1. deriving an open-access database for various scattering environmental conditions at different sites to enable more realistic modelling;

2. giving well-founded guidance on simulation length requirements and the time needed to exclude initial transients, when these realistic conditions are applied, to improve accuracy of numerical simulations.

In order to address these topics, firstly, a database for all significant environmental conditions is derived from real data of the FINO research platforms. In this work, the data source is introduced, the analysis is described, and the resulting distributions and some interesting findings are presented. Secondly, required simulation lengths and times of initial transients are determined. For this purpose, the probabilistic simulation approach and the simulation model are explained. Then, studies of convergence are conducted for the simulation length and the time of initial transients. A monopile and a jacket substructure, FLS and ULS loads, and different wind speeds are considered. Recommendations are summarised. Lastly, the benefits and limitations of the current approach are summarised, and a conclusion is drawn.

\section{Comprehensive database}

\subsection{Raw data}

Environmental conditions can vary significantly among various turbine sites. As these states affect loads, and therefore 
Table 1. Environmental conditions (wind speed $v_{\mathrm{s}}$, significant wave height $H_{\mathrm{s}}$, wave peak period $T_{\mathrm{p}}$, and turbulence intensity TI) of the K13 shallow-water site (UPWIND design basis; Fischer et al., 2010). The wind shear exponent is $\alpha=0.14$, and wind and wave directions are usually set to zero, but scatter plots are available.

\begin{tabular}{lrrrrrrrrrrrrr}
\hline$v_{\mathrm{s}}\left(\mathrm{m} \mathrm{s}^{-1}\right)$ & 2 & 4 & 6 & 8 & 10 & 12 & 14 & 16 & 18 & 20 & 22 & 24 & 26 \\
\hline $\mathrm{TI}(\%)$ & 29.2 & 20.4 & 17.5 & 16.0 & 15.2 & 14.6 & 14.2 & 13.9 & 13.6 & 13.4 & 13.3 & 13.1 & 13.0 \\
$H_{\mathrm{S}}(\mathrm{m})$ & 1.07 & 1.10 & 1.18 & 1.31 & 1.48 & 1.70 & 1.91 & 2.19 & 2.47 & 2.76 & 3.09 & 3.42 & 3.76 \\
$T_{\mathrm{p}}(\mathrm{s})$ & 6.03 & 5.88 & 5.76 & 5.67 & 5.74 & 5.88 & 6.07 & 6.37 & 6.71 & 6.99 & 7.40 & 7.80 & 8.14 \\
\hline
\end{tabular}

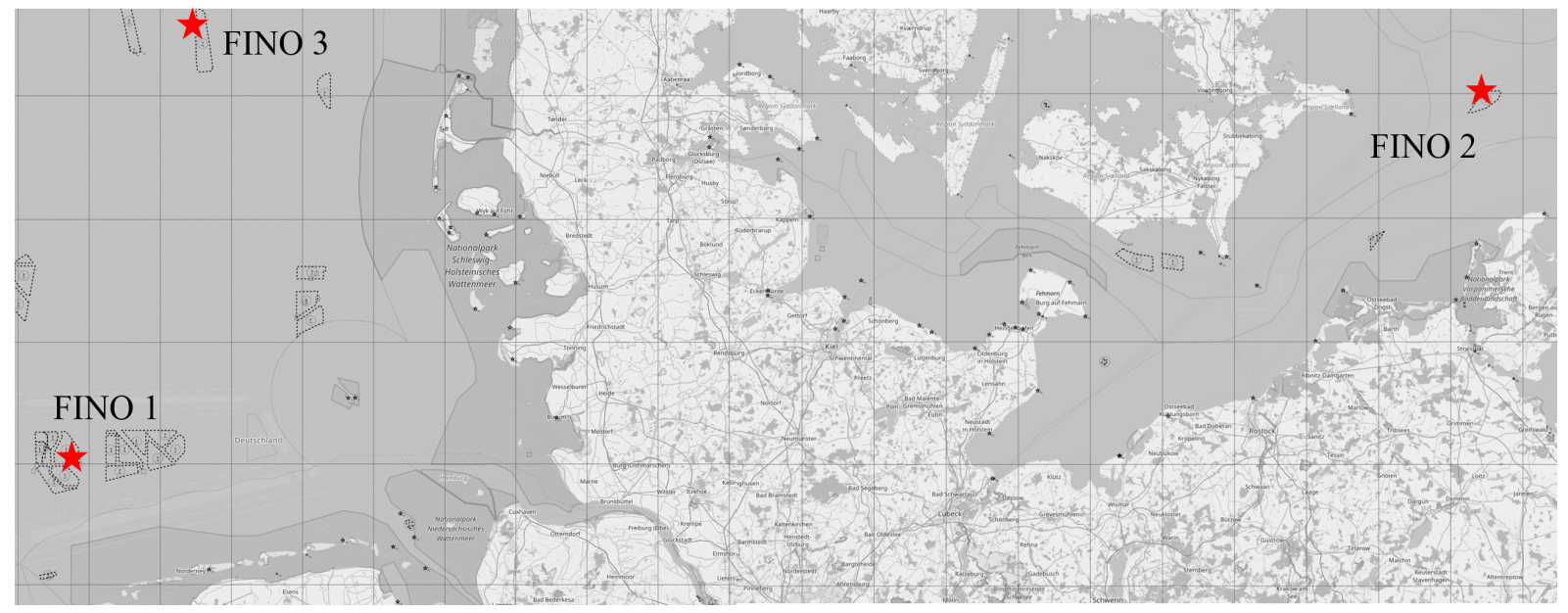

Figure 1. Positions of the three FINO platforms in the North and Baltic Sea, adapted from OpenStreetMap.

the design of offshore wind turbines, precise data of specific turbine location are valuable. Real site data are scarce, which is the reason for the previously mentioned reference databases (Fischer et al., 2010; Hansen et al., 2015; Stewart et al., 2015; Häfele et al., 2017). These databases define conditional, statistical distributions for some of the most important environmental conditions: wind speed and direction, wave height, direction, and peak period. However, other conditions are fixed for each wind speed or are set completely constant. The states of the frequently used UPWIND design basis are summarised in Table 1 as an example.

In this study, scattering conditions are derived directly from offshore measurement data. The raw data are taken from the three FINO platforms, and conditional distributions for the following 13 environmental parameters are determined: wind speed and direction, wave height, peak period and direction, turbulence intensity, wind shear exponent, speed and direction of the sub- and near-surface current, and air and water density. The FINO measurement masts are located in the North Sea and Baltic Sea and are operated on behalf of the German Federal Ministry for the Environment, Nature Conservation, Building and Nuclear Safety (BMUB). ${ }^{1}$ for details. The locations of the three FINO sites are marked in Fig. 1.

\footnotetext{
${ }^{1}$ Raw data of the FINO platforms are freely available for research purposes. See http://www.fino-offshore.de/en/
}

For all three sites, maximum, minimum, mean, and standard deviation values of the wind speed, measured at different heights between 30 and $100 \mathrm{~m}$ above mean sea level, are available for $10 \mathrm{~min}$ intervals. Wind speeds are measured with cup and ultrasonic anemometers. In this study, cup anemometers are used, as these sensors are available at more different heights. For FINO1 and 2, the anemometers are positioned on jibs in secondary wind directions to reduce shadow effects. For FINO3, three anemometers are installed around the mast to minimise shadow effects. Sensors at different heights allow a detailed analysis of shear effects. Wind direction, air pressure, temperature, and humidity are measured at different heights as well. Buoys in the immediate vicinity of the research platforms (about $150 \mathrm{~m}$ ) measure the wave conditions. Mean values of significant wave heights, wave directions, wave peak periods and water temperatures are measured every $30 \mathrm{~min}$. Furthermore, acoustic Doppler current profilers (ADCPs) close to the platforms measure ocean current velocities and directions at different water depths using the Doppler effect of sound waves. The platforms FINO1, 2, and 3 have been measuring continuously since 2004, 2007, and 2009 respectively, resulting in 7 to 13 complete years of measurement data, and enabling at least some long-term predictions. Data of incomplete years are not taken into account in order not to introduce bias due to seasonal effects. 


\subsection{Conditional distributions}

In this work, raw data of the FINO measurement masts are used to set up a database for correlated, scattering environmental conditions. As the post-processing of raw data is time-consuming and it is unnecessary to repeat it each time environmental conditions are used, conditional probability distributions (i.e. $P(Y=y \mid X=x)$, with $X$ being the independent random variable, $Y$ the dependent one, and $P$ the probability function) for environmental conditions are derived to make the database easy to use. Firstly, postprocessing is carried out to identify sensor failures (missing data) and measurement failures (outliers). Missing data are not interpolated but instead left out, in order not to introduce any bias. As sufficient data of proper signal quality are available (e.g. more than 350000 data points for the wind speed even for FINO3), this approach is practicable. Wind speed data are synchronised with the wind direction data. This enables a selection of the anemometer in front of the mast for FINO3. For FINO1 and 2, wind speed values are discarded if the jib is located directly in the tower shadow. The turbulence intensity (TI) can be computed as the quotient of the standard deviation of the wind speed in a $10 \mathrm{~min}$ interval $\left(\sigma_{v}\right)$ and the mean wind speed in this interval $\left(v_{\mathrm{s}}\right)$ according to Eq. (1):

$\mathrm{TI}=\frac{\sigma_{v}}{v_{\mathrm{s}}}$.

For the wind shear, Eq. (2) applies according to the standard of IEC (2005):

$v_{\mathrm{s}}(z)=v_{\mathrm{s}}\left(z_{0}\right) \times\left(\frac{z}{z_{0}}\right)^{\alpha}$,

where $z$ is the height above mean sea level, $z_{0}$ is a reference height, $v_{\mathrm{s}}(z)$ and $v_{\mathrm{s}}\left(z_{0}\right)$ are wind speeds at the specified heights, and $\alpha$ is the wind shear exponent. At the FINO platforms, the wind speed is measured at eight different heights. Therefore, it is possible to determine the wind shear exponent for every $10 \mathrm{~min}$ interval by assuming $z_{0}=90 \mathrm{~m}$ and applying a non-linear regression. The air density can be calculated using Avogadro's law in Eq. (3) and the measurements of humidity $(\phi)$, air pressure $\left(p_{\text {humid }}\right)$, and temperature in degrees Celsius $\left(T_{\text {air }}\right)$ :

$\rho_{\text {air }}=\frac{p_{\text {humid }}}{R_{\text {humid }} T_{\text {air }}}$.

As humid air can be regarded as a mixture of ideal gases, the following equation applies for $R_{\text {humid: }}$

$R_{\text {humid }}=\frac{R_{\text {dry }}}{1-\phi \frac{p_{\text {sat }}}{p_{\text {humid }}}\left(1-\frac{R_{\text {dry }}}{R_{\text {vapour }}}\right)}$,

where $R_{\text {dry }}=287.1 \mathrm{~J} \mathrm{~kg}^{-1} \mathrm{~K}^{-1}$ is the specific gas constant for dry air, $R_{\text {vapour }}=461.5 \mathrm{~J} \mathrm{~kg}^{-1} \mathrm{~K}^{-1}$ for water vapour, and $p_{\text {sat }}$ is the saturation vapour pressure that can, for example, be calculated using the August-Roche-Magnus formula:

$p_{\text {sat }}=6.1094 \mathrm{hPa} \times e^{\frac{17.625 \times T_{\text {air }}}{T_{\text {air }}+243.04}}$.

For the water density, a semi-analytical approach by Millero and Poisson (1981) of the following form is applied:

$$
\begin{aligned}
\rho_{\text {water }}= & A\left(T_{\text {water }}\right)+B\left(T_{\text {water }}\right) S+C\left(T_{\text {water }}\right) S^{1.5} \\
& +D S^{2},
\end{aligned}
$$

where $S$ is the salinity; $T_{\text {water }}$ is the water temperature at the surface; $A, B$, and $C$ are polynomial functions of the water temperature; and $D$ is a constant. As constant salinity is assumed, the water density is a function of the water temperature. For all wave parameters, $3 \mathrm{~h}$ mean values are calculated, as wave conditions stay stationary for a duration of about $3 \mathrm{~h}$ (GL, 2012). For the speeds and directions of suband near-surface currents, measured current values $\left(v_{\mathrm{m}}\right.$ and $\left.\theta_{\mathrm{m}}\right)$ have to be converted in order to separate sub- and nearsurface components. According to, for example, IEC (2009), the following two equations apply for sub- and near-surface currents respectively:

$v_{\mathrm{SS}}(z)=v_{\mathrm{SS}}(0 \mathrm{~m})\left(\frac{d-z}{d}\right)^{\frac{1}{7}}$

and

$v_{\mathrm{NS}}(z)= \begin{cases}v_{\mathrm{NS}}(0 \mathrm{~m})\left(\frac{20 \mathrm{~m}-z}{20 \mathrm{~m}}\right) & \text { for } \quad z<=0 \\ 0 & \text { for } \quad z>0 .\end{cases}$

Here, $v_{\mathrm{SS}}(z)$ and $v_{\mathrm{NS}}(z)$ are the sub- and near-surface current speeds at a position $z$ below the water surface, and $d$ is the water depth. For reasons of clarity, the following notation is introduced: $v_{\mathrm{SS}}(z)=v_{\mathrm{SS}, \mathrm{z}}$. The velocity profiles are shown in Fig. 2. Obviously, the near-surface current does not exist below a reference depth of $20 \mathrm{~m}$. Hence, it is possible to use measurement data of a depth of $20 \mathrm{~m}$ (or more) to directly get the sub-surface direction $\left(\theta_{\mathrm{SS}, 20}=\theta_{\mathrm{m}, 20}\right)$ and to calculate the speed, for example for FINO2 $(d=25 \mathrm{~m})$ :

$v_{\mathrm{SS}, 0}=v_{\mathrm{SS}, 20}\left(\frac{25 \mathrm{~m}-20 \mathrm{~m}}{25 \mathrm{~m}}\right)^{-\frac{1}{7}}$.

For the near-surface current, measurements close to the surface (e.g. $v_{\mathrm{m}, 2}$ ) can be used. However, these measurements include sub- and near-surface components, as shown in Fig. 3.

Therefore, the sub-surface component at $2 \mathrm{~m}$ has to be calculated using Eq. (7), and the sub-surface direction is assumed to be constant over depth $\left(\theta_{\mathrm{SS}, 20}=\theta_{\mathrm{SS}, 2}=\theta_{\mathrm{SS}, 0}\right)$. Then, trigonometrical relationships can be applied to calcu- 


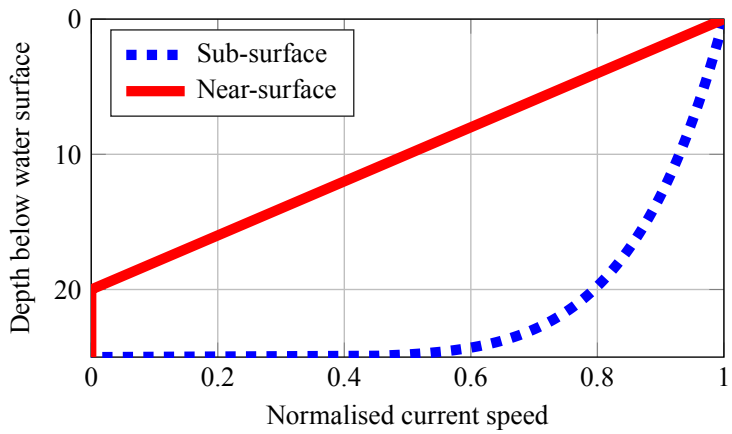

Figure 2. Velocity profiles of the sub- and near-surface currents according to Eqs. (7) and (8) respectively, with a water depth of $25 \mathrm{~m}$ and normalised speeds $\left(v_{\mathrm{SS}, 0}=v_{\mathrm{NS}, 0}=1\right)$.

late the near-surface current at $2 \mathrm{~m}$ :

$$
\begin{aligned}
& v_{\mathrm{NS}, 2}=\sqrt{v_{\mathrm{SS}, 2}^{2}+v_{\mathrm{m}, 2}^{2}-2 v_{\mathrm{SS}, 2} v_{\mathrm{m}, 2} \cos \left(\theta_{\mathrm{m}, 2}-\theta_{\mathrm{SS}, 2}\right)}, \\
& \theta_{\mathrm{NS}, 2}=\theta_{\mathrm{m}, 2}+\arcsin \left(v_{\mathrm{SS}, 2} \frac{\sin \left(\theta_{\mathrm{m}, 2}-\theta_{\mathrm{SS}, 2}\right)}{v_{\mathrm{NS}, 2}}\right) .
\end{aligned}
$$

Lastly, the reference near-surface current $v_{\mathrm{NS}, 0}$ is given by

$$
v_{\mathrm{NS}, 0}=v_{\mathrm{NS}, 2}\left(\frac{20 \mathrm{~m}}{20 \mathrm{~m}-2 \mathrm{~m}}\right) \text {. }
$$

A depth-independent near-surface direction is assumed, and therefore $\theta_{\mathrm{NS}, 0}=\theta_{\mathrm{NS}, 2}$.

After having post-processed the measurement raw data, maximum likelihood estimations are applied to the processed data of the regarded 13 environmental conditions in order to fit several statistical distributions. In addition to unimodal distributions, and if several distinct peaks are distinguishable, multimodal distributions are fitted as well, as it is assumed that the peaks are due to physical phenomena. However, as multimodal approaches have more degrees of freedom, they always fit the data better, even in the case of a physically unimodal shape. Therefore, they have to be chosen with care in order not to fit physically unimodal distributions with multimodal approaches.

Considering the example of wind speed and wave height, it is self-evident that some environmental parameters are conditioned by others, and dependencies have to be defined. For example, the case of a calm sea during a storm is very unlikely. Analysing scatter plots of the environmental inputs and taking a literature review into account, the dependencies in Table 2 are defined, although it is possible to define them differently (see Stewart, 2016), as mainly the correlation is significant, and the determination of cause and effect is secondary.

One of the most common ways to include dependencies in statistical distributions is to split up the data of the dependent parameters into several bins of the independent parameters (e.g. Stewart, 2016; Johannessen et al., 2002; Li et

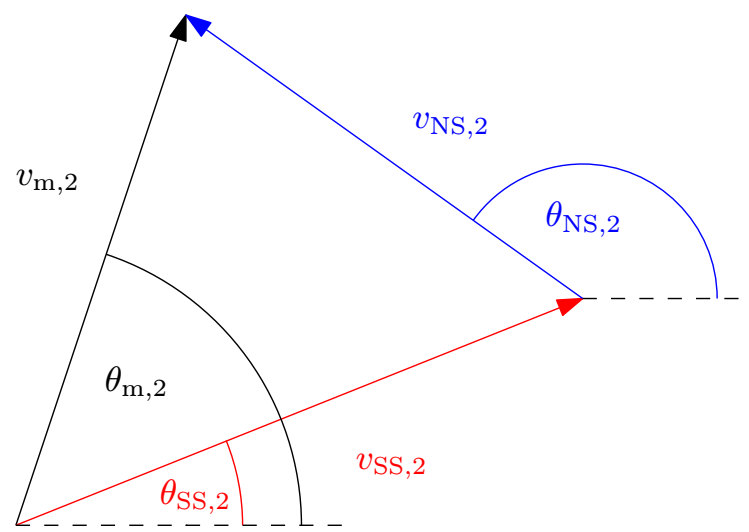

Figure 3. Vectorial analysis of ocean current components at a depth of $2 \mathrm{~m}$ (measured values $(\mathrm{m})$, near- and sub-surface components (NS and SS)).

al., 2015). To illustrate this approach, for example, the wave peak period is fitted in several bins of $0.5 \mathrm{~m}$ wave height (e.g. $P\left(T_{\mathrm{p}}\right)=P\left(T_{\mathrm{p}} \mid 1.5 \mathrm{~m} \leq H_{\mathrm{s}}<2 \mathrm{~m}\right)$ ). The bin widths for the dependent parameters are summarised in Table 2 as well. For highly correlated parameters, an alternative to the binning procedure is to model only the deviation between the parameters. Here, the direction of the near-surface current that is highly dependent on the wind direction is an example. Therefore, by modelling the deviation $\Delta_{\mathrm{NS}}$ according to Table 2, the following applies:

$\theta_{\mathrm{NS}}=\Delta_{\mathrm{NS}}+\theta_{\text {wind }}$

Visual inspections and objective criteria using Kolmogorov-Smirnov tests (KS tests) and chi-squared tests ( $\chi^{2}$ tests) are used to select the best fitting distribution for each environmental condition. Although the KS test is less powerful than other statistical tests, it is still used due to its suitability for small samples (occurring, for example, for dependent variables and high wind speeds), where $\chi^{2}$ tests are not applicable. For one parameter, it is attempted to chose only one distribution for all bins and sites in order to keep the database easy to use. However, as noted in Table 2, in some cases several distributions are selected to increase the accuracy of the fits.

Directional parameters like $\theta_{\text {wind }}$ are treated differently, as classical, parametric distributions can hardly fit several peaks in continuous distributions $\left(0^{\circ}=360^{\circ}\right)$. Therefore, a non-parametric kernel density estimation (KDE) is used to fit directional parameters.

\subsection{Resulting distributions}

In order to establish a full database, statistical distribution and their parameters for all 13 environmental conditions, the three sites and all bins (if necessary) have to be provided. Furthermore, for non-parametric distributions the underlying data are needed. The main ideas are explained here; however, 
Table 2. Dependencies, statistical distributions, and bin widths for environmental conditions derived from FINO1-3 data.

\begin{tabular}{llll}
\hline Parameter & Statistical distributions & Dependencies & Bin sizes \\
\hline Wind speed $\left(v_{\mathrm{S}}\right)$ & Weibull & - & - \\
Wind direction $\left(\theta_{\text {wind }}\right)$ & Non-parametric KDE & Wind speed & $2 \mathrm{~m} \mathrm{~s}^{-1}$ \\
Turbulence intensity $(\mathrm{TI})$ & Weibull, gamma & Wind speed & $2 \mathrm{~m} \mathrm{~s}^{-1}$ \\
Wind shear exponent $\left(\alpha_{\mathrm{PL}}\right)$ & Bimodal normal & Wind speed & $2 \mathrm{~m} \mathrm{~s}^{-1}$ \\
Air density $\left(\rho_{\text {air }}\right)$ & Bimodal log-normal & - & - \\
Significant wave height $\left(H_{\mathrm{S}}\right)$ & Gumbel, Weibull & Wind speed & $2 \mathrm{~m} \mathrm{~s}^{-1}$ \\
Wave peak period $\left(T_{\mathrm{p}}\right)$ & Bimodal Gumbel & Wave height & $0.5 \mathrm{~m}$ \\
Wave direction $\left(\theta_{\text {wave }}\right)$ & Non-parametric KDE & Wave height and wind direction & $1.0 \mathrm{~m}$ and $30^{\circ}$ \\
Water density $\left(\rho_{\text {water }}\right)$ & Trimodal normal & - & - \\
Near-surface current $\left(v_{\mathrm{NS}}\right)$ & Weibull & - & - \\
Sub-surface current $\left(v_{\mathrm{SS}}\right)$ & Weibull, Gumbel & - & - \\
Deviation NS direction $\left(\Delta_{\mathrm{NS}}\right)$ & Bimodal normal & $($ Wind direction and NS direction $)$ & - \\
SS direction $\left(\theta_{\mathrm{SS}}\right)$ & Non-parametric KDE & - & - \\
\hline
\end{tabular}

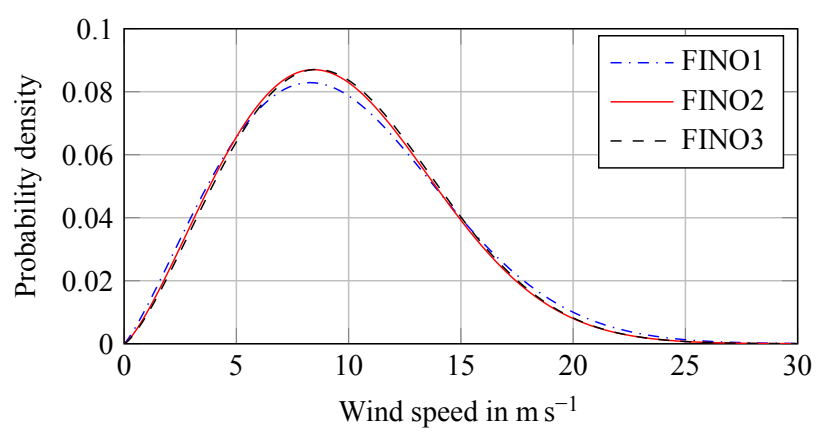

Figure 4. Weibull distributions for the wind speeds for all three sites.

due to the comprehensiveness of the data, detailed and additional information is provided in an easily applicable form, in the Supplement. At this point, only two examples are shown in Figs. 4 and 5.

\subsection{Special findings}

In this section, some noteworthy findings of this database, mainly resulting from the consideration of scattering, are pointed out. Three examples are presented: the importance of wave peak periods, the high scattering of wind shear exponents, and the behaviour of the turbulence intensity.

Wave loads are of particular importance if the wave frequency is close to the first natural frequency of the structure. Standard offshore wind turbines have first bending frequencies of about 0.25 to $0.3 \mathrm{~Hz}$ (Jonkman and Musial, 2010; Popko et al., 2012) corresponding to eigenperiods of less than $4 \mathrm{~s}$. If state-of-the-art databases are used (see Table 1), there will be no resonance. However, real data suggest that resonance effects are problematic even for higher wind speeds, as wave peak periods of less than 4 s occur (see Fig. 6).

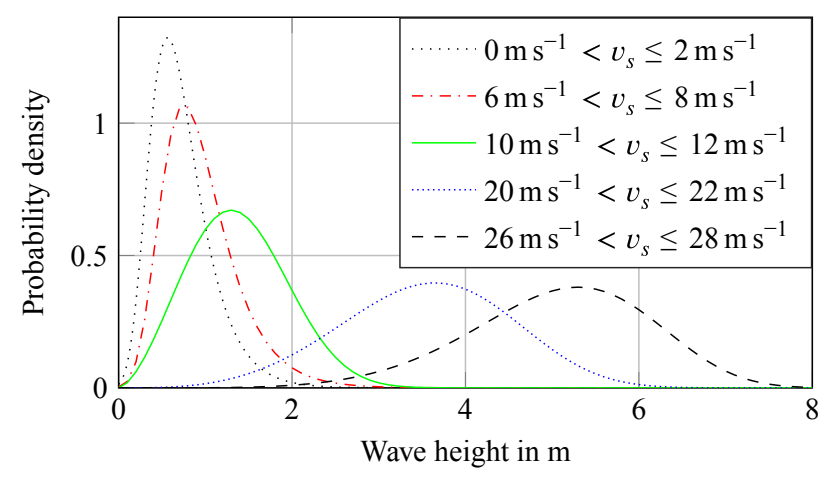

Figure 5. Distribution of the significant wave height for different wind speeds and the FINO1 site. For $v_{\mathrm{s}} \leq 10 \mathrm{~m} \mathrm{~s}^{-1}$, Gumbel distributions are applied. For higher wind speeds, Weibull distributions fit the data more accurately.

Concerning the wind shear exponent, in the standards and most current databases (e.g. GL, 2012; Fischer et al., 2010), constant values for all wind speeds are proposed. However, this assumption is a massive simplification. Ernst and Seume (2012) showed that the wind shear exponent significantly depends on the wind speed. Here, it is shown (see Fig. 7) that it does not only vary between wind speeds but also scatters remarkably within each bin as well, and might even be negative.

For the turbulence intensity, this database reveals that state-of-the-art approaches are mainly conservative, as too high turbulence intensities are assumed. This is shown in Fig. 8, where the turbulence intensity for all three sites is compared to a standard database (Fischer et al., 2010) and to current standards (IEC, 2009). All three sites exhibit similar mean turbulence intensities and 90th percentile values $\left(Q_{0.9}\right)$. For the comparison with literature values, the 90th percentile is of importance, as standards require simulations with this percentile value. However, even for the 90th per- 


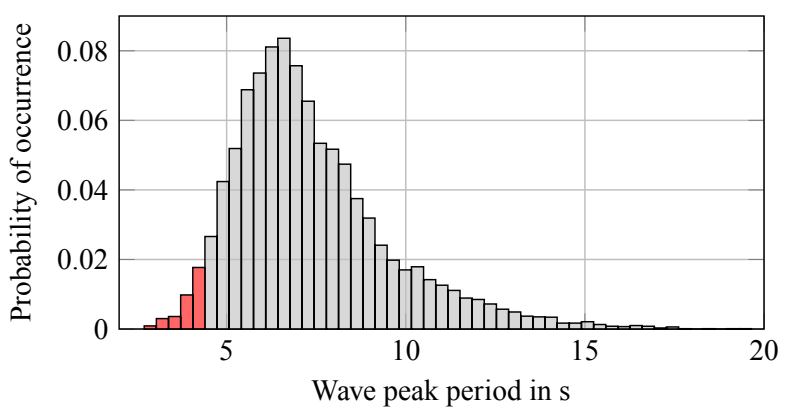

Figure 6. Probability distribution of the wave peak period for $v_{\mathrm{S}}=11-13 \mathrm{~m} \mathrm{~s}^{-1}$ for the FINO3 site.

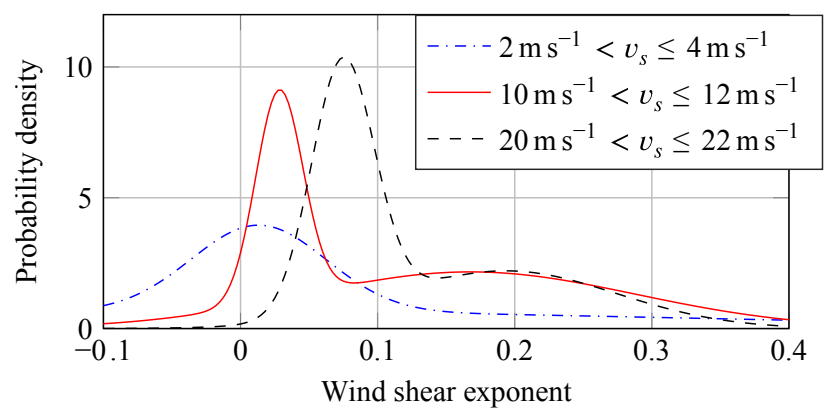

Figure 7. Distribution of the wind shear exponent for different wind speeds for the FINO2 site.

centile, the UPWIND database is very conservative. The least conservative case (category C) in IEC (2009) fits the $Q_{0.9}$ values relatively well, but it predicts slightly higher turbulence intensities for wind speeds above about $10 \mathrm{~m} \mathrm{~s}^{-1}$. Considering the fact that using the 90th percentile is a conservative assumption and that the measurements include some wake effects due to wind farms near to all measurement masts, it can be concluded that state-of-the-art assumptions for turbulence intensities are probably unnecessarily conservative. The wake effects are depicted in Fig. 9, where turbulence intensity measurements of FINO1 from 2011 to 2016 are shown. In this period, the wind farm Alpha Ventus was operating on the east side of FINO1. Therefore, west wind leads to free stream conditions and east wind to wake conditions. Obviously, free stream conditions lead to even lower turbulence intensities, whereas wake conditions increase the turbulence, especially for smaller wind speeds, as also detected by Hansen et al. (2012).

\section{Simulation assistance}

In the previous section, a comprehensive database for scattering environmental offshore conditions was developed. However, even with realistic input parameters the accuracy of numerical simulations is significantly influenced by constraints like their lengths and the time eliminated to exclude ini-

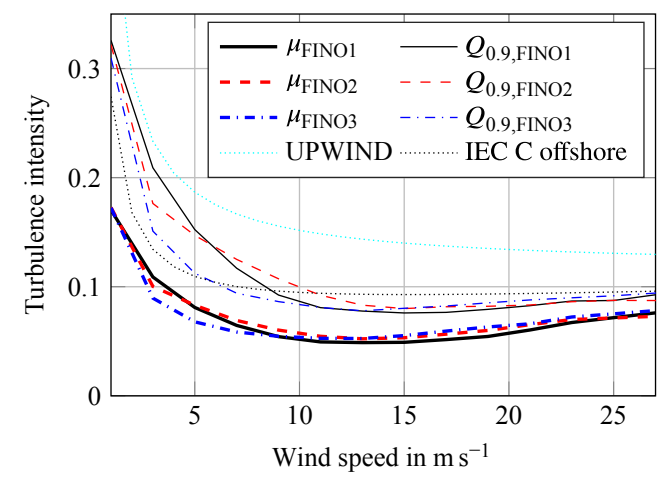

Figure 8. Turbulence intensity (mean value and 90th percentile $\left(Q_{0.9}\right)$ ) for different wind speeds compared to the literature.

tial transients. Therefore, in this section, efficient simulation lengths and times of initial transients for varying wind speeds and different types of loading and substructures are determined. This is achieved by analysing the convergence of relevant quantities (i.e. FLS and ULS loads). Before conducting these studies, the overall probabilistic simulation approach is explained, as it differs from the approach in the standards. Subsequently, the utilised simulation model and the chosen environmental conditions are briefly presented.

\subsection{Probabilistic simulation approach}

For the design of offshore wind turbines, several design load cases (DLC1.1 to 8.3) have to be simulated according to the standards (IEC, 2009). These load cases cover ultimate and fatigue loads during power production, idling and fault conditions, and several special cases like start-up or shut-down. Stochastic inputs for turbulent wind and irregular wind are included. Nevertheless, the DLCs remain quasideterministic, as environmental conditions like turbulence intensities and wind shear do not scatter. In order to guarantee safe designs despite the deterministic approach, several ULS load cases, covering extreme environmental conditions (e.g. DLC1.3 for turbulence or DLC1.5 for wind shear), are needed.

In this work, statistically scattering environmental conditions are applied, and therefore a probabilistic simulation approach is used. This probabilistic approach differs from the deterministic load-case-based approach. For the probabilistic approach or "real-life" approach, it is not necessary to simulate any load cases of extreme environmental conditions (e.g. DLC1.3 to 1.6), but the use of scattering conditions leads directly to simulations that represent the real lifetime of the turbine (without fault, start-up, or other special situations). Hence, simulations (e.g. 10000 simulations) cover a realistic period of power production and idling, leading to about 2.3 months of turbine lifetime (for 10000 simulations). As environmental conditions scatter, effects like high turbulences, extreme wind shear, high waves, small wave periods, 


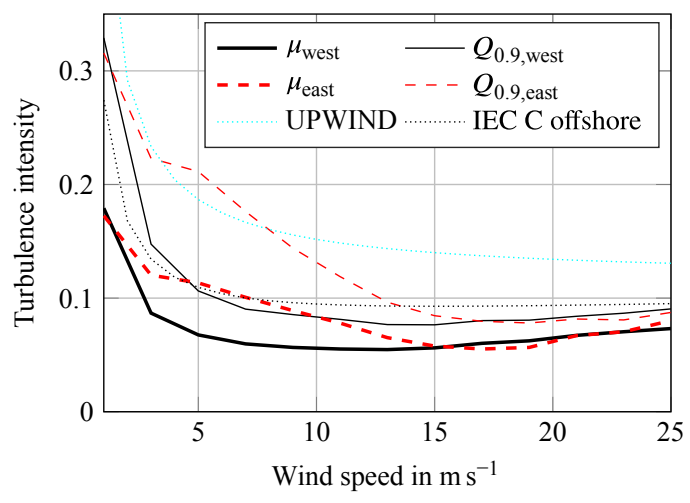

Figure 9. Shadow effects on the turbulence intensity for FINO1 and free stream (western) and wake (eastern) conditions.

and others are covered and do not have to be considered separately. Load cases are not simulated explicitly, but are covered implicitly by conducting probabilistic simulations.

That is why the two approaches do not differ significantly for FLS. The "real-life" approach covers DLC 1.2 and 6.4. For ULS, the "real-life" approach covers all power production cases (DLC 1.1-1.6) and DLC 6.1 by applying scattering environmental conditions. As the "real-life" approach cannot simulate 20 years of turbine lifetime (or even a return period of 50 years), a load extrapolation, as required for DLC 1.1, is needed in order to calculate an ULS design. However, this extrapolation is not needed here, as it does not influence the investigated simulation constraints.

As common in academia, only power production and idling is simulated. Fault cases, start-up, etc. are not taken into account due to several reasons. Firstly, at least for the jacket, fault cases are less relevant (Vemula et al., 2010). Secondly, these load cases are very controller and design dependent and need special treatment (e.g. there is no need of removing initial transients for start-up load cases). Thirdly, this work is not intended to calculate exact fatigue damages or ultimate loads for the whole turbine lifetime, as no turbine design or optimisation is done. The exclusion of some load cases does not affect the recommendations on simulation constraints that are given for power production and idling conditions. As there is no need of exact FLS and ULS lifetime loads in this study, an assessment of the probabilistic approach concerning accordance with the standards is neither conducted nor needed, but this would be valuable for further applications of probabilistic approaches.

\subsection{Simulation setup}

As environmental conditions vary for various turbine sites, a database being used for the studies of convergence has to be chosen. The basis developed in this work is appropriate, and the FINO3 site is chosen. Some conditions, like air and water density, are kept fixed, as it was shown that their variation is of minor importance (Hübler et al., 2017). An attempt is made to keep the convergence study as simple as possible, and to focus on the most relevant parameters. Hence, for the probabilistic approach, statistically scattering values according to the determined distributions of wind speed and direction, wave height, direction and period, turbulence intensity, and wind shear exponent are used in all simulations. In addition, the following assumptions are made for all simulations:

- The turbulent wind field is computed according to the Kaimal model and using the software TurbSIM (Jonkman, 2009) with a different wind seed for each simulation.

- Irregular waves are calculated according to the JONSWAP spectrum using varying wave seeds for all simulations.

- Soil conditions of the OC3 model (Jonkman and Musial, 2010) are applied.

- The current, second-order and breaking waves, wave spreading effects, marine growth, local vibration effects of braces, joint stiffnesses, and degradation effects are neglected.

The time domain simulations of the convergence study are conducted using the aero-servo-hydro-elastic simulation framework FASTv8 (Jonkman, 2013). A soil model (Häfele et al., 2016) applying linearised soil-structure interaction matrices enhances this code. The NREL $5 \mathrm{MW}$ reference wind turbine (Jonkman et al., 2009) with two different substructures is investigated: Firstly, the OC3 monopile (Jonkman and Musial, 2010) and secondly, the OC4 jacket (Vorpahl et al., 2013). The outcomes of the FAST simulations are, inter alia, time series of forces, moments, and stresses for each element of the substructure.

Since the convergence of fatigue and ultimate loads is investigated in the next step, the calculation concept of these two loads is briefly explained.

For the jacket, the procedure of the fatigue analysis in accordance with DNV (2010) is the following: for each connection of each joint ( $\mathrm{K}$ joints, $\mathrm{Y}$ joint, butt welds, etc.), eight hotspot stresses around the circumference of the intersection have to be calculated using the time series. The needed stress concentration factors (SCFs) depending on the joint geometry are calculated according to Appendix B of DNV (2010). The fatigue damage is calculated with a fatigue limit of $52.6 \mathrm{MPa}$ at $10^{7}$ cycles. This corresponds to the DNV-GL $\mathrm{S}-\mathrm{N}$ curve 90 (for cathodic protection) as used in the original design (Vemula et al., 2010). For all stresses, rainflow counting evaluates the stress cycles. As recommended by the current standards, the conservative damage accumulation according to the Palmgren-Miner rule is assumed using a slope of the $\mathrm{S}-\mathrm{N}$ curve of 3 before and 5 after the fatigue limit for both substructures. The separated fatigue calculation (and summation over all simulations) for each connection of each 
joint is necessary, as damages in each connection and joint are different for each simulation, and the highest values do not always occur in the same joint (for example due to the probabilistic variation of the wind direction). Finally, the decisive damage for the jacket is the highest accumulated value of all connections of all joints.

For the monopile, the fatigue procedure is similar, but is done according to European Committee for Standardization (2010b), where a detail of $71 \mathrm{MPa}$ for transverse butt welds and an additional reduction due to the size effect $(t>25 \mathrm{~mm})$ is recommended. Differing from the recommendations in European Committee for Standardization (2010b), the same slopes of the $\mathrm{S}-\mathrm{N}$ curves as for the jacket are used.

For the ULS analysis, maximum stresses are decisive and extracted from the time series. For the monopile, European Committee for Standardization (2010a) is used to analyse the plastic limit state, cyclic plasticity limit state, and buckling limit state (LS1-3). For the jacket, NORSOK N-004 is applied for tubular members and joints, which takes combined axial, shear, bending, and hydrostatic loadings into account. In both cases, the yield stress is $355 \mathrm{MPa}$.

Additionally, ultimate limit state proofs for the foundation piles are performed including axial and lateral soil proofs according to GEO2 (DIN 1054, 2010) and a plastic limit state proof (LS1) for the steel pile below mudline. Especially for the monopile, the last proof might be decisive as the bending moment frequently reaches its maximum below mudline. For all ULS proofs, utilisation factors, being the percentage of the maximum loads, are the outcomes.

\subsection{Simulation length}

The simulation length significantly influences the overall computing time of the load assessment. However, there is no conclusive consensus concerning the length needed. Current standards recommend, for example, $10 \mathrm{~min}$ or $1 \mathrm{~h}$ calculations. The offshore oil and gas industry prefers simulation lengths of $6 \mathrm{~h}$ to cover all low-frequency hydrodynamic effects.

The use of $10 \mathrm{~min}$ simulations can potentially reduce the computing time by a factor of about 36 compared to $6 \mathrm{~h}$ simulations. Hence, a study of convergence for bottom-fixed offshore wind turbines is conducted here. For floating wind turbines, it is referred to Stewart (2016), who showed that for floating structures all physical effects can be covered with 10 min simulations.

The presented outcomes of this study focus on the monopile substructure, but a jacket is analysed as well and results (not shown) are generally comparable. For several wind speed bins, 500 simulations with a total length of $10 \mathrm{~h}$ are conducted. As the initial transient behaviour is analysed subsequently, a clearly sufficient time, being discarded to exclude the initial transients, of $4 \mathrm{~h}$ is chosen. With elimination of these $4 \mathrm{~h}$ of initial transients, the total length of $10 \mathrm{~h}$ reduces to a maximum available length (simulation length) of
$6 \mathrm{~h}$ for the convergence study. In a first step, the convergence of FLS loads is analysed. Afterwards, the ULS case is investigated.

The procedure to calculate the mean fatigue damage for each wind speed bin is the following: from the basis of the 500 ten-hour simulations having different random seeds and varying environmental conditions, 500 cases are selected (with replacement). For each simulation, the fatigue damage is calculated and weighted with the simulation length. The mean value of all cases is calculated. This procedure is repeated 10000 times (bootstrapping) to assess the associated uncertainty.

Figure 10 displays the normalised mean fatigue damages for different wind speeds and simulation lengths between $10 \mathrm{~min}$ and $6 \mathrm{~h}$. The values are normalised with the $6 \mathrm{~h}$ values, and error bars show the $\pm \sigma$ confidence intervals (68\%) that are estimated using a bootstrap procedure with 10000 resamplings.

It is apparent that due to scattering environmental conditions and the limited number of simulations the uncertainty is relatively high. A detailed investigation of the fatigue load uncertainty, when scattering environmental conditions are applied, is valuable but out of the scope of this work (see Sect. 4). Nevertheless, from Fig. 10 it is apparent that there are no pronounced trends for changing simulation lengths. A slight increase in fatigue loads for higher simulation lengths might be suspected given the fact that such behaviour was observed for floating substructures by Stewart (2016). In order to focus on the simulation length effects, the variation of environmental conditions is neglected in a second step (only varying random seeds). This reduces the uncertainty, making it possible to clearly identify a slight increase in FLS loads of about $5 \%$ for higher simulations lengths (see Fig. 11, non-merged case). However, as shown by Stewart (2016) for floating substructures, the increasing fatigue loads are not due to any physical effect (all important low-frequency effects of waves are already covered by 10 min simulations), but can be explained by the effect of unclosed cycles in the rainflow counting. Cycles that are not completed at the end of the simulation are approximated by counting them as half cycles. The longer the simulation, the less influential this approximation is, as the number of half cycles compared to the number of full cycles reduces. A quite straightforward approach to reduce the problem of half cycles is to merge several shorter simulations (e.g. $10 \mathrm{~min}$ simulations) into a longer one (e.g. $6 \mathrm{~h}$ simulation). This means fatigue damages are not calculated for each time series separately but rather for longer time series consisting of several shorter ones that are just appended to each other. It is possible to either append different 10 min time series to each other or each time series is duplicated and appended several times to itself. If scattering environmental conditions are assumed, in some simulations, fairly different load levels occur. In these cases, load levels of the simulations might not fit, and additional cycles can be introduced by merging different time series, leading to 


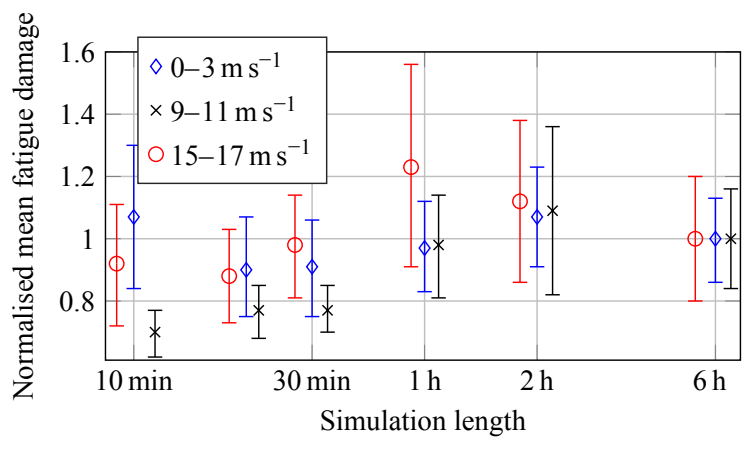

Figure 10. Normalised mean fatigue damage (500 simulations) for increasing simulation lengths and different wind speeds.

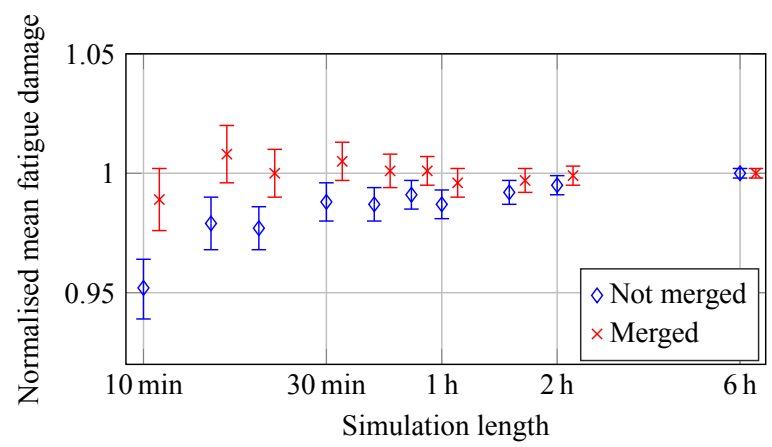

Figure 11. Normalised mean fatigue damage (500 simulations) for increasing simulation lengths and $v_{\mathrm{S}}=9-11 \mathrm{~m} \mathrm{~s}^{-1}$. Environmental conditions are kept constant to demonstrate the effect of merging time series more clearly.

unreasonably increased fatigue damages. Merging each time series with itself guarantees fitting load levels. A downside of this is that the computing time of the post-processing is slightly increased. The effect of merging several shorter simulations with themselves to generic and repetitive $6 \mathrm{~h}$ time series (e.g. each $10 \mathrm{~min}$ time series is duplicated 36 times and is appended to itself to create a $6 \mathrm{~h}$ time series) is demonstrated in Fig. 11. It can be seen that the simulation error of about $5 \%$ too low FLS loads for non-merged 10 min simulations can be compensated for by merging time series in the post-processing.

For the ULS loads, the calculation procedure is similar. From the basis of the 500 ten-hour simulations, 500 cases are selected (with replacement). The maximum value of all simulations is taken as decisive utilisation factor. This procedure is repeated 10000 times (bootstrapping) to assess the associated uncertainty.

The convergence is shown in Fig. 12. Obviously, ULS loads are higher for longer simulations. Again, this increase is not due to any physical phenomenon, but a result of different overall computing times. Clearly, 500 ten-minute simulations should not be compared to 500 six-hour simulations but

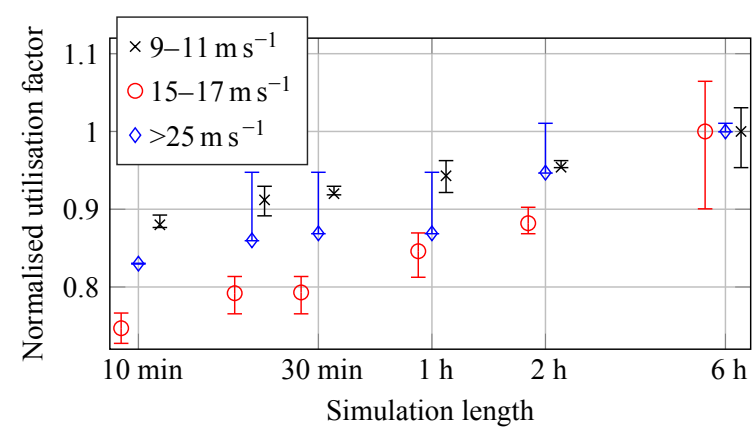

Figure 12. Normalised mean ULS utilisation factor (500 simulations) for increasing simulation lengths and different wind speeds.

instead to about 14 six-hour simulations (Haid et al., 2013). Therefore, in a second step, the ULS calculation procedure is slightly adapted. Now, 500 cases are only selected for $10 \mathrm{~min}$ simulations. For all other simulations length, the number of cases is reduced to keep the over simulation length constant at $5000 \mathrm{~min}$ (i.e. 250 cases for $20 \mathrm{~min}$ simulation, for example). This comparison is displayed in Fig. 13 and makes clear that ULS loads do not depend on the simulation length but instead on the overall computing time. A second fact being visible in Fig. 13 are the higher uncertainties for longer simulation lengths. Since $10 \mathrm{~min}$ simulations lead to a higher number of cases than $6 \mathrm{~h}$ simulations for the same total length (i.e. 500 and 14), shorter simulations better cover rare cases, and therefore scattering environmental conditions leading to less uncertainty.

After all, the investigations of this section suggest that simulations of $10 \mathrm{~min}$ length are sufficient independent of the type of load or investigated substructure, or wind speed. At this point, it has to be noted that only two types of substructures are analysed and environmental conditions typical for the North Sea. For significantly different substructures or locations, the validity might be limited. Notwithstanding the above, for ULS loads, the same overall time has to be compared in order to achieve reliable results. By keeping the simulation length short, more simulations can be conducted in the same overall computing time leading to a better convergence of ULS loads. For FLS loads, simulation errors due to the simulation length can be reduced by merging the time series.

\subsection{Initial transients}

For the analysis of the simulation length, the first $4 \mathrm{~h}$ of each simulation were discarded to guarantee a steady-state operation of the turbine. However, removing $4 \mathrm{~h}$ of initial transients and only using 10 min of simulation is computationally very expensive. Therefore, the convergence of FLS and ULS loads with respect to the time of initial transients is analysed. As initial conditions, like an initial rotor speed, influence the initial transient behaviour (Haid et al., 2013), initial 


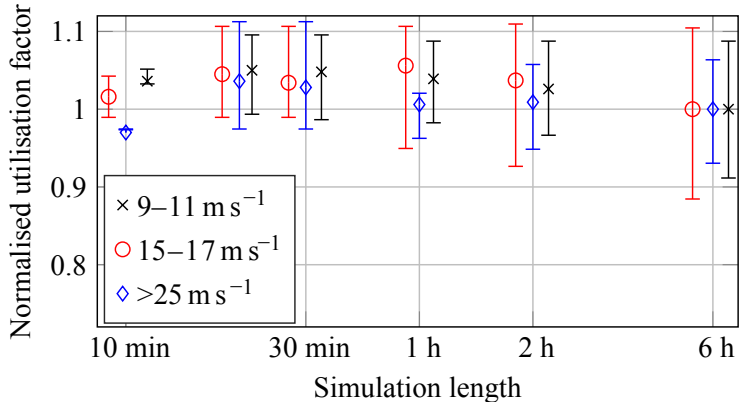

Figure 13. Normalised mean ULS utilisation factor for increasing simulation lengths (constant overall length of $500 \times 10$ min, leading to 500 to 14 simulations) and different wind speeds.

rotor speeds and blade pitches depending on the wind speed are set here. These initial conditions are quasi-static states determined using prior simulations.

As the initial transient behaviour is affected by the type of substructure and the load condition, the time that has to be removed is analysed in each wind speed bin for FLS and ULS loads and for both types of substructures separately. Commonly, time series are investigated to estimate times of initial transients (Zwick and Muskulus, 2015). Although this is a straightforward approach, here it is considered to not be expedient. For a fatigue assessment, the convergence of the fatigue damage has to be analysed, and for the ULS analysis, maximum loads or utilisation factors have be considered.

For each wind speed bin, 10000 simulations for the monopile and 500 for the jacket were conducted according to the simulation setup in Sect. 3.2. This means that each simulation has its own random seed for irregular waves and turbulent wind, and in addition, different wind speeds and directions, wave heights, directions and periods, turbulence intensities and wind shear exponents according to the FINO3 data are applied. The high and unequal number of simulations is needed to exclude effects of the number of simulations, mentioned in the previous section and addressed in Sect. 4, as well as possible. For the monopile, each simulation at operating conditions is $900 \mathrm{~s}$ long $(600 \mathrm{~s}$ simulation length plus $300 \mathrm{~s}$ of initial transients) and $1800 \mathrm{~s}$ at idling conditions. When the turbine is idling, the aerodynamic damping is lower, leading to more pronounced initial transients. For the jacket, all simulations are $720 \mathrm{~s}$ long. Using this simulated database, it is possible to analyse the effect of different initial simulation times removed on the fatigue damage and utilisation factors in order to determine optima. The analysed simulation length is kept constant at $600 \mathrm{~s}$, while the removed length varies between 0 and $300 \mathrm{~s}$ ( $1200 \mathrm{~s}$ for idling; $120 \mathrm{~s}$ for the jacket).

Figure 14 displays the convergence of the fatigue damage of the monopile substructure at operating conditions. Here, $300 \mathrm{~s}$ or $120 \mathrm{~s}$ values are used as a reference, the so-called "converged value". The $10 \mathrm{~h}$ simulations in Sect. 3.3 were

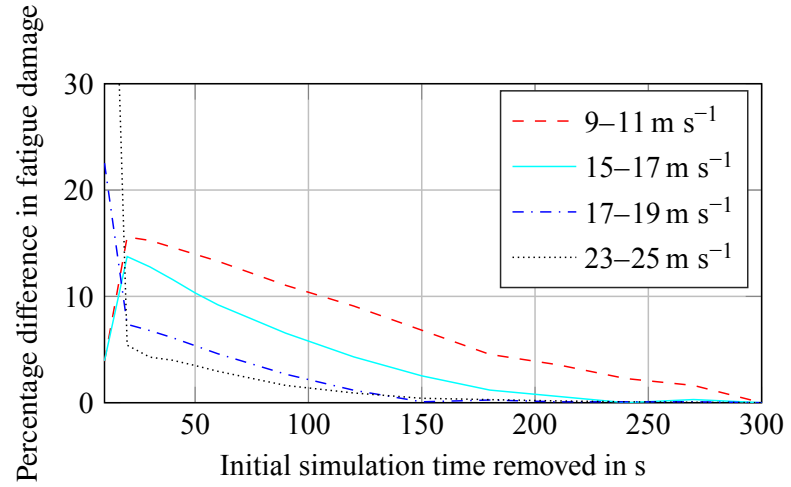

Figure 14. Initial transient behaviour of the operating wind turbine with a monopile substructure for different wind speeds. Percentage difference in the fatigue damage compared to the "converged" value (300s).

used determine these values, where the error due to initial transients can be neglected and is much smaller than the error due to the number of simulations. For idling conditions (not shown), the initial transient behaviour takes longer, as the aerodynamic damping is lower. For the same reason, the transients are shorter for higher wind speeds. For the jacket substructure displayed in Fig. 15, the transients decay much faster in all wind speed bins. As jackets are less influenced by wave loads, being not always aligned with the wind, the aerodynamically marginally damped side-to-side modes are less excited, leading to a shorter transient behaviour. This interpretation is supported by the fact that for the jacket, idling conditions, where the hydrodynamic behaviour dominates, have shorter initial transients.

The convergence of ULS utilisation factors for both substructures is shown in Figs. 16 and 17. It becomes apparent that initial transients are short independent of the type of substructure and wind speed. The cycles with high amplitudes occurring at the beginning of each simulation are damped out within a few seconds, and hence are not influencing the ULS behaviour. More problematic are less damped cycles with smaller amplitudes leading to the previously presented, higher times of initial transients for FLS loads.

The recommended times that should be discarded to exclude initial transients for both substructures, being always a compromise between computing time and accuracy (here, errors below 5\%), are summarised in Table 3 . It has to be mentioned that the general validity is limited, as these times of initial transient might vary, for example, for different aero-elastic codes, numerical solvers, time constants of the aero-elastic models, or substantially different substructures. For example, jackets for $10 \mathrm{MW}$ turbines might behave differently due to larger diameters of legs and braces increasing wave effects. However, for similar applications (e.g. FASTv8, NREL $5 \mathrm{MW}$ turbine, OC3 monopile, or OC4 jacket) that are not rare in academia (e.g. 
Table 3. Recommended times that should be discarded to exclude initial transients for simulations with OC4 jacket and OC3 monopile substructures for different wind speeds to achieve errors below $5 \%$.

\begin{tabular}{lrrrrrrrrrrrrrr}
\hline$v_{\mathrm{s}}$ in m s & Case & $<3$ & $3-5$ & $5-7$ & $7-9$ & $9-11$ & $11-13$ & $13-15$ & $15-17$ & $17-19$ & $19-21$ & $21-23$ & $23-25$ & $>25$ \\
\hline Monopile & \multirow{2}{*}{$\mathrm{FLS}$} & $720 \mathrm{~s}$ & $240 \mathrm{~s}$ & $240 \mathrm{~s}$ & $240 \mathrm{~s}$ & $240 \mathrm{~s}$ & $240 \mathrm{~s}$ & $240 \mathrm{~s}$ & $150 \mathrm{~s}$ & $120 \mathrm{~s}$ & $60 \mathrm{~s}$ & $60 \mathrm{~s}$ & $60 \mathrm{~s}$ & $360 \mathrm{~s}$ \\
Jacket & & $40 \mathrm{~s}$ & $30 \mathrm{~s}$ & $50 \mathrm{~s}$ & $40 \mathrm{~s}$ & $50 \mathrm{~s}$ & $50 \mathrm{~s}$ & $50 \mathrm{~s}$ & $50 \mathrm{~s}$ & $50 \mathrm{~s}$ & $60 \mathrm{~s}$ & $50 \mathrm{~s}$ & $50 \mathrm{~s}$ & $10 \mathrm{~s}$ \\
\hline Monopile & \multirow{2}{*}{$\mathrm{ULS}$} & $<10 \mathrm{~s}$ & $<10 \mathrm{~s}$ & $<10 \mathrm{~s}$ & $<10 \mathrm{~s}$ & $10 \mathrm{~s}$ & $10 \mathrm{~s}$ & $10 \mathrm{~s}$ & $10 \mathrm{~s}$ & $10 \mathrm{~s}$ & $10 \mathrm{~s}$ & $10 \mathrm{~s}$ & $10 \mathrm{~s}$ & $<10 \mathrm{~s}$ \\
Jacket & & $<10 \mathrm{~s}$ & $20 \mathrm{~s}$ & $20 \mathrm{~s}$ & $20 \mathrm{~s}$ & $20 \mathrm{~s}$ & $20 \mathrm{~s}$ & $20 \mathrm{~s}$ & $20 \mathrm{~s}$ & $20 \mathrm{~s}$ & $20 \mathrm{~s}$ & $20 \mathrm{~s}$ & $20 \mathrm{~s}$ & $<10 \mathrm{~s}$ \\
\hline
\end{tabular}

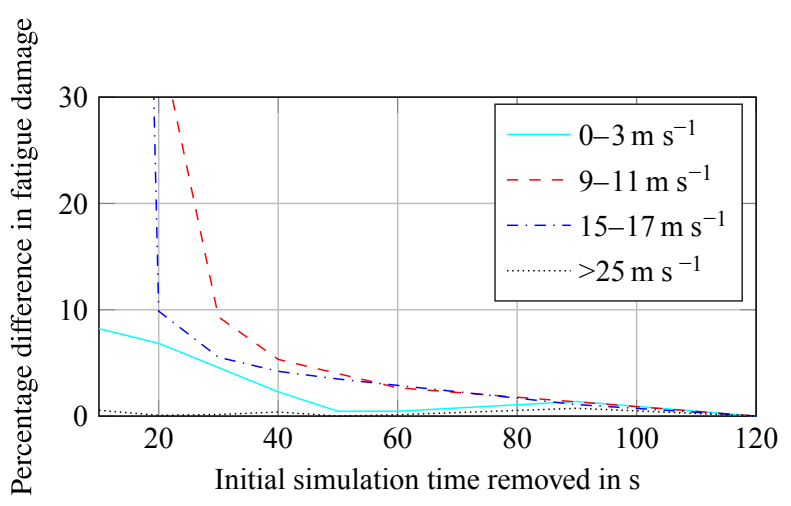

Figure 15. Initial transient behaviour of the wind turbine with a jacket substructure for different wind speeds. Percentage difference in the fatigue damage compared to the "converged" value (120 s).

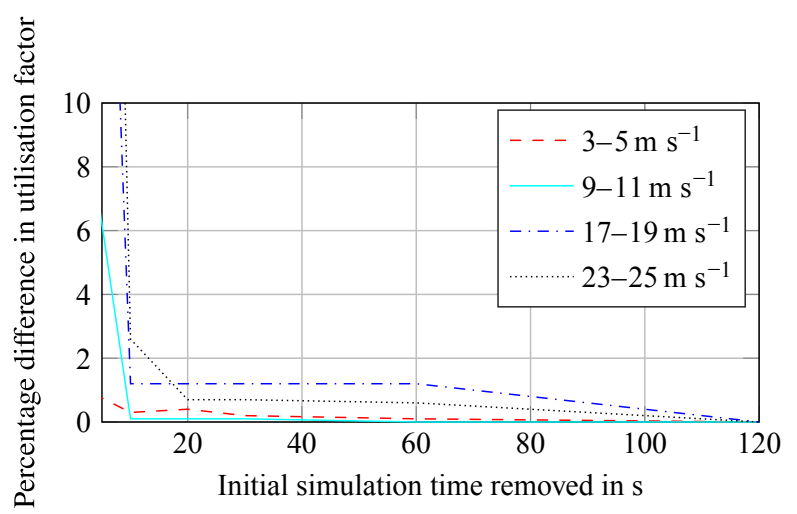

Figure 16. Initial transient behaviour of the wind turbine with a monopile substructure for different wind speeds. Percentage difference in the utilisation factor (ULS) compared to the "converged" value (120s).

Zwick and Muskulus, 2015 or Morató et al., 2017), the given values represent a well-founded guidance for simulation setups. Furthermore, these results should sensitise the research community to the problem of initial transients especially in the case of fatigue. For fatigue, the time of initial transients might be higher than frequently presumed in the literature. This is due to weakly damped cycles with small amplitudes that cannot directly be identified when looking at time series.

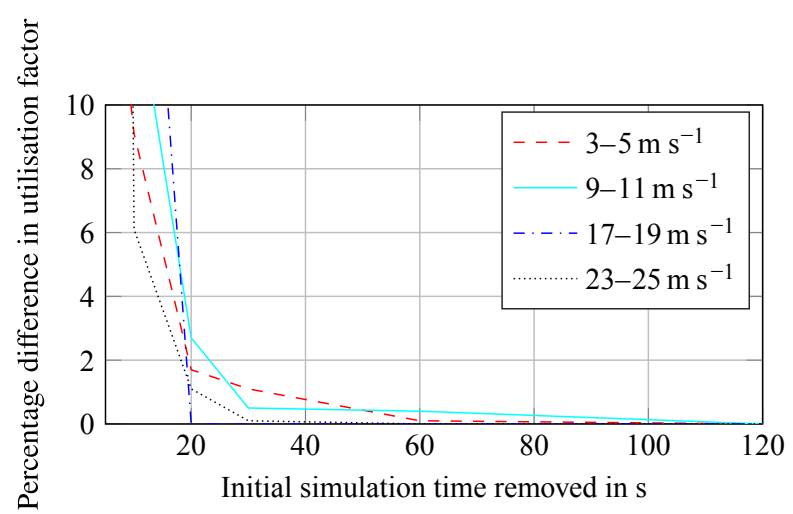

Figure 17. Initial transient behaviour of the wind turbine with a jacket substructure for different wind speeds. Percentage difference in the utilisation factor (ULS) compared to the "converged" value (120s).

\section{Benefits and limitations}

The benefit of the current work is twofold. Firstly, a comprehensive database for scattering environmental conditions was set up, which is freely available and easy to use. Secondly, two simulation constraints (simulation length and time of initial transients) were analysed, and well-founded recommendations are given.

The main advantages over existing databases are the following: the database covers several different sites situated in different oceans. It has to be admitted that the sites are fairly similar, as they are all in shallow-water conditions. Additionally, the database contains statistical distribution for much more environmental conditions than existing ones. As was shown, for example, by Hübler et al. (2017) that not only main conditions like the wind speed are influencing the dynamic behaviour of offshore wind turbines, knowledge of additional parameters is beneficial. Current databases consist frequently of raw data that need to be post-processed, which is a time-consuming process. Here, on the one hand, easily applicable statistical distributions are given. On the other hand, the complexity of dependent environmental conditions is still covered by utilising conditional distributions and multimodal and non-parametric approaches. In contrast to many existing databases, the raw data are of good quality. 
For example, wind speeds are measured at heights comparable to hub heights of current turbines, and there is no need for extrapolations, as is the case for buoy measurements. Still, more data would be valuable in order to achieve more reliable distributions in high-wind-speed bins that rarely occur. After all, the developed database is capable of improving offshore wind turbine modelling by providing more realistic inputs for simulations in academia, where real site data are scarce. One example of improved offshore wind turbine modelling is given in Sect. 3.3 and 3.4. The inclusion of probabilistic inputs leads to a significant and realistic increase in fatigue damage scattering requiring high numbers of simulations. Hence, deterministic inputs underestimating this scattering can lead to biased fatigue values. Detailed analyses of the effect of scattering environmental conditions on fatigue damage, and therefore of the needed number of simulations, are part of upcoming work of the authors.

Concerning the second benefit, the simulation constraints, it has to be kept in mind that not only realistic modelling but also small simulation errors are important in order to model accurately. In this context, the chosen simulation length and time of initial simulation transients matter. So far, these values are frequently chosen without profound knowledge. Some approaches to gain a deeper insight into these constraints (Stewart, 2016; Zwick and Muskulus, 2015) concentrate on simulation lengths or specific types of substructures and are not taking realistically scattering environmental conditions into account. In this work, the scattering of the conditions is addressed and different bottom-fixed substructures are analysed. This enables recommendations for simulation lengths and times of initial transients depending on the wind speed, the type of substructure, and the considered load case (ULS or FLS). However, the general validity of the current results has to be slightly restricted, as only one design of each type of substructure was investigated. Therefore, the initial transient behaviour might be slightly different for significantly different designs. Furthermore, for the time being removed to exclude initial transients, the values might also differ between different simulation codes and are only tested for the FASTv8 code. Different numerical solver or time constants of the aero-elastic models might also influence the time of initial transients. Nevertheless, even in these cases, firstly, the given recommendations can be regarded as a well-founded starting point for further investigations. Secondly, and even more important, they clarify the challenge of a well-founded choice.

\section{Conclusions}

This work aims to help future simulation work to be more realistic and accurate. In order to achieve this objective, a freely available and comprehensive database for scattering environmental conditions was set up. This database consists of conditional statistical distribution for many parameters and can be applied without further post-processing. All needed information (statistical distribution and their parameters) is given in the Supplement. In academia, this database enables simulations with probabilistic environmental conditions making them more realistic. For industry purposes, this work might lead to a reconsideration of the current practice. This study shows that the use of deterministic values that are either only dependent on the wind speed (e.g. turbulence intensity) or even totally constant (e.g. wind shear) does not represent realistic offshore conditions. However, for a well-founded reconsideration of the current practice, a detailed assessment of probabilistic approaches compared to deterministic loadcase-based ones is needed.

Additionally, scientifically sound recommendations are given for the choice of simulation lengths and times to be removed to exclude initial transients. Simulation lengths of $10 \mathrm{~min}$ are generally sufficient, and can even help to reduce uncertainties. However, in the case of FLS loads, times series should be merged, and for ULS situations, the overall computing time has to be kept constant. Recommendations concerning the initial transients have to be handled with care due to limitations of the general validity. The values are summarised in Table 3 and can help to improve the accuracy of simulations, and to reduce computing times. It should be noted that a partly significantly longer initial transient behaviour compared to values in the literature was detected. Literature values are mainly based on educated guesses so far.

An enlargement of the current database to include additional offshore sites, other types or designs of substructures, or investigations for other simulation codes and numerical solver would be definitely valuable to increase the general validity. Furthermore, even for the utilised FAST code, additional investigations concerning the amount of eigenmodes representing the substructure would be beneficial, as a reduction of retained eigenmodes might reduce the time of initial transients.

Data availability. The raw data are taken from the FINO platforms - operated on behalf of the Federal Ministry for the Environment, Nature Conservation, Building and Nuclear Safety (BMUB) - and are freely available for research purposes (http://www. fino-offshore.de/en/). The derived database, consisting of statistical distribution for 13 partly dependent environmental conditions and three offshore sites, is freely available. All needed information concerning the statistical distribution and their parameters is given in the Supplement.

\section{The Supplement related to this article is available online at https://doi.org/10.5194/wes-2-491-2017-supplement.}


Competing interests. The authors declare that they have no conflict of interest.

Acknowledgements. We gratefully acknowledge the financial support of the Lower Saxony Ministry of Science and Culture (research project VENTUS EFFICIENS, FKZ ZN3024) and the European Commission (research project IRPWIND, funded from the European Union's Seventh Framework Programme for research, technological development, and demonstration under grant agreement number 609795) that enabled this work. This work was supported by the compute cluster which is funded by Leibniz Universität Hannover, the Lower Saxony Ministry of Science and Culture (MWK), and the German Research Foundation (DFG).

The publication of this article was funded by the open-access fund of Leibniz Universität Hannover.

Edited by: Michael Muskulus

Reviewed by: two anonymous referees

\section{References}

Cheng, P. W.: A reliability based design methodology for extreme responses of offshore wind turbines, DUWIND Delft University Wind Energy Research Institute, the Netherlands, 2002.

Det Norske Veritas (DNV): Fatigue design of offshore steel structures, Recommended practice DNV-RP-C203, available at: https://rules.dnvgl.com/docs/pdf/DNV/codes/docs/2010-04/ RP-C203.pdf (last access: October 2017), 2010.

Det Norske Veritas (DNV): Design of floating wind turbine structures, Offshore Standard DNV-OS-J103, available at: http://rules.dnvgl.com/docs/pdf/DNV/codes/docs/2013-06/ OS-J103.pdf (last access: October 2017), 2013.

DIN - Normenausschuss Bauwesen: Subsoil - Verification of the safety of earthworks and foundations - Supplementary rules to DIN EN 1997-1, DIN 1054, available at: https://www-1 perinorm-1 com-100000boj285b.shan02.han.tib. eu/document.aspx (last access: October 2017), 2010.

Ernst, B. and Seume, J. R.: Investigation of site-specific wind field parameters and their effect on loads of offshore wind turbines, Energies, 5, 3835-3855, 2012.

European Committee for Standardization: Eurocode 3: Design of steel structures - Part 1-6: Strength and stability of shell structures, EN 1993-1-6, Brussels, Belgium, 2010a.

European Committee for Standardization: Eurocode 3: Design of steel structures - Part 1-9: Fatigue, EN 1993-1-9, Brussels, Belgium, 2010b.

Fischer, T., de Vries, W., and Schmidt, B.: Upwind Design Basis, Endowed Chair of Wind Energy (SWE) at the Institute of Aircraft Design Universität Stuttgart, Germany, 2010.

Germanischer Lloyd (GL): Guideline for the Certification of Offshore Wind Turbines, Offshore Standard, Hamburg, Germany, 2012.

Häfele, J., Hübler, C., Gebhardt, C. G., and Rolfes, R.: An improved two-step soil-structure interaction modeling method for dynamical analyses of offshore wind turbines, Appl. Ocean Res., 55, 141-150, 2016.
Häfele, J., Hübler, C., Gebhardt, C. G., and Rolfes, R.: Effcient Fatigue Limit State Design Load Sets for Jacket Substructures Considering Probability Distributions of Environmental States, The 27th International Ocean and Polar Engineering Conference, 2530 June 2017, San Francisco, USA, 167-173, 2017.

Haid, L., Stewart, G., Jonkman, J., Robertson, A., Lackner, M., and Matha, D.: Simulation-length requirements in the loads analysis of offshore floating wind turbines, 32nd International Conference on Ocean, Offshore and Arctic Engineering, 9-14 June 2013, Nantes, France, 1-10, ASME, 2013.

Hansen, K. S., Barthelmie, R. J., Jensen, L. E., and Sommer, A.: The impact of turbulence intensity and atmospheric stability on power deficits due to wind turbine wakes at Horns Rev wind farm, Wind Energy, 15, 183-196, 2012.

Hansen, M., Schmidt, B., Ernst, B., Seume, J., Wilms, M., Hildebrandt, A., Schlurmann, T., Achmus, M., Schmoor, K., Schaumann, P., Kelma, S., Goretzka, J., Rolfes, R., Lohaus, L., Werner, M., Poll, G., Böttcher, R., Wehner, M., Fuchs, F., and Brenner, S.: Probabilistic Safety Assessment of Offshore Wind Turbines, Leibniz Universität Hannover, Hannover, Germany, 2015.

Hübler, C., Gebhardt, C. G., and Rolfes, R.: Hierarchical Four-Step Global Sensitivity Analysis of Offshore Wind Turbines Based on Aeroelastic Time-Domain Simulations, Renew. Energ., 111, 878-891, 2017.

International Electrotechnical Commission (IEC): Wind turbines part 1: Design requirements, International standard IEC 61400-1, Geneva, Switzerland, 2005.

International Electrotechnical Commission (IEC): Wind turbines part 3: Design requirements for offshore wind turbines, International standard IEC 61400-3, Geneva, Switzerland, 2009.

Johannessen, K., Meling, T. S., and Hayer, S.: Joint distribution for wind and waves in the northern north sea, Int. J. Offshore Polar, 12, 1-8 2002.

Jonkman, B. J.: TurbSim user's guide: Version 1.50, National Renewable Energy Laboratory, Golden, Colorado, USA, 2009.

Jonkman, J.: The New Modularization Framework for the FAST Wind Turbine CAE Tool, 51st AIAA Aerospace Sciences Meeting, including the New Horizons Forum and Aerospace Exposition, 7-10 January 2013, Dallas, Texas, USA, 1-26, 2013.

Jonkman, J., Butterfield, S., Musial, W., and Scott, G.: Definition of a 5 MW Reference Wind Turbine for Offshore System Development, National Renewable Energy Laboratory, Golden, Colorado, USA, 2009.

Jonkman, J. M. and Musial, W.: Offshore Code Comparison Collaboration (OC3) for IEA Task 23 Offshore Wind Technology and Deployment, National Renewable Energy Laboratory, Golden, Colorado, USA, 2010.

Kost, C., Mayer, J. N., Thomson, J., Hartmann, N., Senkpiel, C., Philipps, S., Nold, S., Lude, S., and Schlegl, T.: Stromgestehungskosten erneuerbare Energien, Fraunhofer-Institut für solare Energiesysteme ISE, Freiburg, Germany, 2013.

Li, L., Gao, Z., and Moan, T.: Joint distribution of environmental condition at five european offshore sites for design of combined wind and wave energy devices, J. Offshore Mech. Arct., 137, 031901, https://doi.org/10.1115/1.4029842, 2015.

Millero, F. J. and Poisson, A.: International one-atmosphere equation of state of seawater, Deep-Sea Res. Pt. A, 28, 625-629, 1981. 
Morató, A., Sriramula, S., Krishnan, N., and Nichols, J.: Ultimate loads and response analysis of a monopile supported offshore wind turbine using fully coupled simulation, Renew. Energ., 101, 126-143, 2017.

Popko, W., Vorpahl, F., Zuga, A., Kohlmeier, M., Jonkman, J., Robertson, A., Larsen, T. J., Yde, A., Saetertro, K., Okstad, K. M., Nichols, J., Nygaard, T. A., Gao, Z., Manolas, D., Kim, K., Yu, Q., Shi, W., Park, H., and Vásquez-Rojas, A.: Offshore Code Comparison Collaboration Continuation (OC4), Phase 1 - Results of Coupled Simulations of an Offshore Wind Turbine With Jacket Support Structure, The Twenty-second International Offshore and Polar Engineering Conference, International Society of Offshore and Polar Engineers, 17-22 June 2012, Rhodes, Greece, 337-346, 2012.

Prognos AG and Fichtner: Kostensenkungspotenziale der OffshoreWindenergie in Deutschland, Stiftung Offshore-Windenergie, Berlin, Germany, 2013.

Stewart, G. M.: Design Load Analysis of Two Floating Offshore Wind Turbine Concepts, PhD thesis, University of Massachusetts, Massachusetts, USA, 2016.

Stewart, G. M., Lackner, M., Haid, L., Matha, D., Jonkman, J., and Robertson, A.: Assessing fatigue and ultimate load uncertainty in floating offshore wind turbines due to varying simulation length, Safety, Reliability, Risk and Life-Cycle Performance of Structures and Infrastructures, CRC Press, London, UK, 239246, 2013.
Stewart, G. M., Robertson, A., Jonkman, J., and Lackner, M. A.: The creation of a comprehensive metocean data set for offshore wind turbine simulations, Wind Energy, 19, 1151-1159, 2015.

Vemula, N. K., de Vries, W., Fischer, T., Cordle, A., and Schmidt, B.: Design Solution for the UpWind Reference Offshore Support Structure - Deliverable D4.2.5 (WP4: Offshore Foundations and Support Structures), Rambøll Wind Energy, Esbjerg, Denmark, 2010.

Vorpahl, F., Popko, W., and Kaufer, D.: Description of a basic model of the "UpWind reference jacket" for code comparison in the OC4 project under IEA Wind Annex 30, Fraunhofer IWES, Bremerhaven, Germany, 2013.

Zwick, D. and Muskulus, M.: The simulation error caused by input loading variability in offshore wind turbine structural analysis, Wind Energy, 18, 1421-1432, 2015. 